\section{SOI: $\underline{1.1 / \mathrm{TAS}}$ DOI: $\underline{10.15863 / \mathrm{TAS}}$ International Scientific Journal Theoretical \& Applied Science}

p-ISSN: 2308-4944 (print) ｅ-ISSN: 2409-0085 (online)

Year: $2015 \quad$ Issue: 03 Volume: 23

Published: $30.03 .2015 \quad$ http://T-Science.org
EInur Latif oglu Hasanov Corresponding member of International Academy of Theoretical and Applied Sciences, Ph.D. postgraduate Institute of Local-lore of Ganja Branch Azerbaijan National Academy of Sciences, Ganja, Azerbaijan 1-hasan@,hotmail.com

SECTION 12. Geology. Anthropology.

Archaeology.

\title{
MIRZA SHAFI VAZEH AND GANJA. CREATION OF M.S. VAZEH AS ETHNOGRAPHICAL AND LITERARY SOURCE
}

\author{
Abstract: In this scientific work for the first time has been systematic investigated the main moments of \\ activities of the famous poet and philosopher Mirza Shafi Vazeh on the basis of new literary and historical facts. \\ Key words: Ganja, Mirza Shafi Vazeh, Azerbaijan, poet and philosopher, XIX century. \\ Language: German \\ Citation: Hasanov EL (2015) MIRZA SHAFI VAZEH AND GANJA. CREATION OF M.S. VAZEH AS \\ ETHNOGRAPHICAL AND LITERARY SOURCE. ISJ Theoretical \& Applied Science 03 (23): 5-9. \\ Soi: http://s-o-i.org/1.1/TAS*03(23)2 Doi: crossef http://dx.doi.org/10.15863/TAS.2015.03.23.2

\section{MIRSÄ SCHÄFY WASEH UND GÄNDSCHÄ \\ (ERBE DER M.S. WASEH ALS ETHNOGRAPHISCHE UND LITERARISCHE QUELLE)}

Die Zusammenfassung: In dieser wissenschaftlichen Arbeit zum ersten Mal wurde systematisch untersucht die wichtigsten Momente der Tätigkeit des berühmten Dichters und Philosophen Mirsä Schäfy Waseh, auf der Grundlage des neuen literarischen und historischen Fakten.

Die Schlüsselwörter: Gändschä, Mirsä Schäfy Waseh, Aserbaidschan, Gelehrten und Dichter, XIX Jahrhundert.

\section{Die einführung}

Die Natur von Aserbaidschan unterscheidet sich mit Vielfältigkeit, darum nennt man Aserbaidschan die touristische Perle des Südkaukasus. Die Hälfte des Territoriums besteht aus wunderschönen Niederungen und von Kür-Gewässer. Aserbaidschan liegt im Herzen des südlichen Kaukasus und zieht immer auf sich die Aufmerksamkeit. Wie früher gibt es jetzt auch keinen Menschen, der dieses alte Land mit reicher Geschichte und freundlichem Klima nicht liebt. Die Berge des großen und kleinen Kaukasus umgeben das Land. Es ist vom Kaspischen Meer umgespult. Das Klima von Aserbaidschan ist einzigartig und weich für den Pflanzenbau.

Die reiche Pflanzenwelt des Landes erobert mit seiner Aussicht, Natur, Landschaften blühenden Wiesen und Wäldern die Herzen der strengen Reisenden. Herrliche Berge und Wasserfälle, die die Ruhe und die Wonne jedem schenken vollenden das Bild. Die Pflanzenwelt Aserbaidschans unterscheidet sich durch verschiedenartige Pflanzenarten, deren Zahl auf diesem Boden gegen vier tausend Arten macht, unter deren auch sehr seltene Pflanzen gibt. Die gabenreiche Natur Aserbaidschans vereinigt die Züge des Kaukasus, Asiens und Europas.

Aber der Hauptreichtum des Landes besteht in seinem Unikum, besonderem Kolorit, seiner Einzigartigkeit. Aserbaidschan könnte man für sein Naturreichtum und klimatische Vielfältigkeit vom ganzen Herzen als eine Stelle bezeichnen, die in sich das Beste was auf der Erde gibt vereinigt hat.

Im Mittelalter gegründete Gändschä wurde zu einem der Hauptzentren von Aserbaidschan. Diese große Stadt wurde die Heimat der begabten aserbaidschanischen Söhne und Töchter. Einen großen Beitrag zur Literatur des Mittellalters leisteten Nisami Gändschäwi mit seinem Poem "Chamse" und Mähsäti Gändschäwi.

Der berühmte Aserbaidschanische Aufklärer Mirsä Schäfi Waseh (1794-1852) war auch aus 
Gändschä. Die am Fuß vom Kleinkaukasus, im Brustkorbe des Flusses Gändschä, im Herzen Aserbaidschans liegende Stadt Gändschä befindet sich auf dem großen aus China nach Europa führenden Seidenweg.

Gändschä war im Laufe der Jahrhunderte und ist jetzt das Zentrum der Wissenschaft, Kultur, Industrie und des Handels im Kaukasus und in Aserbaidschan.

Gändschä schenkte einen der ersten vaterländischen Orientalisten M. D. Toptschubaschow. Man kann auch viele Dichter, die in Gändschä lebten, nennen, zum Beispiel Mirsä Mechti Hadschi, Molla Abdulla, Hadschi Abbas, Haki. Schöne Gebirge bewachen Stadt. Jeden Morgen weidet Gändschä mit ihrer Widerspiegelung im Wasser von Göygöl. Gändschä erinnert sich auch an die Zeit, als die schönen Toren der Stadt ihre Ruhe bewachten. Es gibt hier auch bei der Einfahrt in die Stadt das Nisami - Mausoleum, das seine herliche Aussicht erhalten hat.

Die Kunst des Teppichknüpfens ist eine der bedeutenden Erfolge von Ostvölkern. Die Herstellung der Teppiche erschien im I Jahrhundert vor unserem Zeitalter in Aserbaidschan. Aber erschien das Teppichknüpfen wie eine selbständige Kunst im ersten Mittelalter. Die Stadt Gändschä, die 4000 Jahre alt hatte, unterscheidet sich mit seinem, schnellentwickelden Teppichknüpfen. Gändschä ist eine älteste Ecke der Kultur unseres Landes. In alten Gändschä, die das Vaterland von berühmten aserbaidschanischen Dichter und Gelehrten Scheich Nisami Gändschäwi ist, wurden sehr seltsame und einzigartige Teppiche hergestellte. In der Stadt Gändschä, die reiche Traditionen hatte, die Teppiche mit typischen unterschiedlichen Eigenschaften hergestellt. Deshalb die Gändschänischen Teppiche (oder Gändschäs-Kasach) bilden eine Gruppe von aserbaidschanischen Teppichen. In den Perioden von XIX Jahrhundert wurden in Gändschä örtliche Arten von Teppichen geknüpft. Diese Teppiche, die von der Bevölkerung geknüpft wurden, unterschieden sich mit ihren Besonderheiten: Die örtlichen Meister gebrauchen die butaförmigen Ornamente meisterhaft, die Gändschänischen Teppiche unterschieden sich mit ihrem hohen Knüpfen, diese Arten von Teppichen unterschieden sich mit ihrer eng Zeichnung und ein wenig Minderheit ( $25 \times 30)$, für die Färbung einen großen Teil der Teppiche wurden mit den grellen Farben gebraucht und kompositionen sind verständlich und einfach. Man muss es betonen, dass die Gändschänischen Teppiche immer mit ihren hohen Qualitäten und künstlichen Eigenschaften hoch geschätzt wurden. Und deshalb wurden einige herrliche Gändschänischen Teppiche im XIX Jahrhundert auf den bedeutenden Ausstellungen demonstriert. In der Gegenwart werden die Gändschänischen Teppiche in den maßgeblichen
Museen, oder in den persönlichen Kollektionen bewahren.

\section{Erde der Mirsä Schäfy Waseh}

Mirsä Schäfy Waseh wurde in den 1794 Jahren in Gändschä. Waseh bekam eine zeitlang Religionsbildung. Da er Interesse zur weltlichen Wissenschaft und Aufklärung hatte, sagten die Gläubiger in Gändschäs Medrese ihn zu unterrichten ab. Indessen folge verließ Waseh Medrese. Nach der Entlassung von Medrese begann er selbstständig zur Vermehrung seiner Kenntnisse und den Weltkreis durch sein fleißiges Üben. Im XIX Jahrhundert hat man seine Gedichte mit großem Erfolg angenommen und danach brachte seinen Autor in die erste Reihe unter den Ostklassikern. Obwohl aus dem Original seiner aserbaidschanischen und persischen Gedichte keine geblieben waren und trotz seines größten Ruhmsin Europa und Rußland, konnten seine Werke im Nahen Orient und in seiner Heimat Aserbaidschan nicht verbreitet werden. Mirsä Schäfy Waseh, wurde in der Kärbälaji Sadyks Mauerfamilie geboren. Sein Vater K.Sadyk war in dem Palast von Dschavad Chan beschäftigt. Als Kind hat Waseh seinen Vater verloren und bei Person Hadschi Abdulla gewohnt. Kärbälaji Sadyk wollte, dass sein Sohn geistliches Studium bekam. Das gute Beherrschen des Persischen gab ihm Möglichkeit die Werke von Klassiker wie Chayyam, Nisami, Sady, Hafis näher kennenzulernen. Mit Hilfe Hadschi Abdulla und für die Regierung der Dörfer und Guttümer von Dschavad Chans Tochter Puste chanym wurde Mirsa Schäfy zum Beruf Mirsa (Schreiber) festgestellt. Deshalb wurde er Mirsa Schäffy genannt, verbunden mit seinem neuen Beruf. 1826 wurde die Tochter Dschavad Chans Puste chanym wegen des Krieges zwischen Russland und Iran gezwungen, mit ihrem Bruder Ugurlu chan nach Iran zu fliehen. Und Mirsä Schäffy blieb arbeitslos. Zu dieser Zeit starb auch Hadschi Abdulla. Obwohl Mirsa Schäfy allein in schwerer Lage blieb, verlor seine Begeisterung nicht. Er begann als Schreiber und Lehrer zu arbeiten. In Gändschä begann er in der Moschee Schach Abbas die Schüler zum Sauberschreiben und Lernen zu lehren. Einer von seinen Schülern war Mirsa Fätäly Achundov. Mirsä Schäffy ist 1840 aus Gändschä nach Tbilisi gefahren. Im November dieses Jahres wurde er mit Hilfe.

Mirsä Fätäly Achundov in die Schule als Lehrer von Aserbaidschanisch und Phersisch ernannt. Er hat bis Ende des Jahres 1846 in Tbilisi gelebt. Nach der Rückkehr aus Tbilisi hat er ein Gedicht „Abschied“ geschrieben. Im Jahre 1844 schuf er in Tbilisieinen philosophischen Literaturzirkel Divani - Hikmet"(Versammlung von Weisen). In „Divani-Hikmät“ las man zuerst 
Gedichte und dann führte man Besprechungen. Damals haben an diesen Gedichtsbesprechungen Abbasqulu Aga Bakichanov, Mirsa Fätäly Achundov, Dichter Nädshmi, Mirsa Häsän, Hadschi Abdulla, Mirsa Jusif, Widadi, Hadschi Jusif teilgenommen. Dank dieser Versammlungen hatte Mirsa Schäffy viele hervorragendste Wissenschaftler und Künstler kennengelernt. Einer von Versammlungsmitgliedern war Friedrich von Bodenstedt, der im Jahre 1819 - in Deutschland, im Rand - Niedersachsen im Vorort Pajne bei Hannover geboren. Bodenstedt war jüdischer Herkunft. Er wurde an besten Deutschlands Universitäten - Göttingen, München, Berlin Studium gemacht. Im Jahre 1841 ist Bodenstedt nach Moskau gekommen und dort hat er mit Erziehung des Sohns vom Fürst Michajil Galizin beschäftigt. Nach der Einladung des Hauptgenerals Neidtgardt ist er im Jahre 1844 nach Tbilisi gekommen, begann er hier zur Lehrertätigkeit. Er hat von Mirsa Schäffy persische Sprache gelernt. Mirsä Schäffy hat mit der Hilfe von Bodenstedt die Werke von West Europaklassiker kennengelernt. Im Jahre 1846 ist Bodenstedt nach Deutschland zurückgekehrt. Mirsä Schäffy Vaseh ist im Jahre 1846 nach Gändschä zurückgekehrt und wurde hier in einer neuen Schule als Lehrer ernannt. Er beschäftigte sich nicht nur mit seiner Lehrertätigkeit, sondern schuf Gedichte auch. 1850 ist er nach Tbilisi zurückgekehrt und hat er dort als Lehrer gearbeitet. Am 28.11.1852 wurde er in Tbilisi gestorben und beerdigt. Mirsä Schafys begräbniss befindet sich hinter dem Botanischen Garten. Mirsä Schäfys Gedichte, die im Russischen und Westeuropäischen Sprachen veröffentlicht waren, haben kein Original im Persischen und Aserbaidschanischen. Friedrich von Bodenstedt hat dem Manuskript dieser Gedichte nach Deutschland gebracht.

Nach der Bekanntschaft mit Bodenstedt liess Mirsä Schäfy im Jahre 1844 in Tbilisi seine Gedichte ihm schreiben. Außerdem hatte M. Schäfy das Manuskript seiner Gedichtsammlung dem Bodenstedt geschenkt. Bodenstedt schrieb selbst, dass „Er schenkte mir das Heft unter dem Titel "Der Schlüssel der Weisheit". Im Vorwort dieses Heftes schrieb M. Schäfy so, "Ich, Mirsä Schäfy schenke ihm mein Werkversammlung - Ode, Gasel, Strophen und Mesnevi“. Als Bodenstedt in Tbilisi war, hat er die Gedichte von Mirsa Schäfy gesammelt und im Jahre 1846 nach Deutschland mitgebracht und dort diese Gedichte übersetzt und veröffentlicht. Im Jahre 1851 wurde „Tausend ein Tag im Orient" von Bodenstedt und danach ein kleines Büchlein „Die Lieder des Mirsa Schäfy“ veröffentlicht. Dieses Büchlein hat dem Dichter einen Weltruhm gebracht. Mirsa Schäfys Gedichte wurden bald in ganzen Westeuropa verbreitet, man übersetzte sie in verschiedene Sprachen-Englische, Französische, Italienische, Schwedische, Norvegische, Holländische, Polnische und sogar in uralte Judische (Hibridische). Diese Gedichte erweckten in Russland ein grosses Interesse. Die Übersetzung und die Veröffentlichung dieser „Lieder“ gehörte dem Dichter Michail Larinovitsch Michajilov dem Freund von N.G.Tschernyschevsky (1826-1865). Nach Michailov hatten diese Gedichte V. M. Markov, M.Rams, N. Ejfert und andere übersetzt. Nachdem Tolstoj mit diesem Gedicht bekannt wurde, bewertete er diese Gedichtesehr hoch. Mirsa Schäfys Gedichte wurden in Deutschland 1868 sechsmal, im Jahre 1876 zwölfmal veröffentlicht. Bis 1922 wurde es 169 Mal veröffentlicht. Solche eine umfangreiche Veröffentlichung gelang keinem anderen Dichter. Als Bodenstedt sah, dass "Lieder“" in Westeuropa und Russland sehr berühmt waren, lehnte ab 1873 das Dichtertum seines Lehrers ab und stellte sich als Autor dieser Gedichte vor. Orientalist Adolf Berdshe verneinte völlig auch $\mathrm{M}$. Schäffy als Dichter nach der Meinung von Transkaukasiens Scheychkhulislams Mulla Ähmäd Säljani. Bodenstedt betrug die literarische Gesellschaft genannt zuerst Gedichte Mirsa Schäfys zu Seinem und verband das mit seinen Osteindrücken. Er nehme Mirsa Schäfy als Pseudonym aus dem Orient. Danach fand sich Bodenstedt in Westeuropa Helfer und wurde Mirsä Schäfys Name lange Zeit fast vergessen. Obwohl bei der Bekanntmachung Mirsä Schäfys Gedichte in Europa der Verursacher Bodenstedt war, brachte ihn sein dieser literarischer Stahl zum Schmach und Schande. Wenn er Gedichte unter Mirsä Schäfys Namen veröffentlichte, wurde er sehr berühmt, aber umgekehrt unter seinen Namen erweckte er Unglaube. Es muss bemerkt sein, dass Bodenstedt in Mirsa Schaffys Lieder manche ihm nicht gehörende Schreiben hinzugefügt hatte.

\section{Der abschluss}

1. In manchen Quellen basiert man diese Gazel falsch auf Mulla Wali Widadi. Diese Gedichte, die zum kleinen Teil Mirsä Schäfys Schaffen gehört, wurden ersten Mal von Salman Mumtas geoffenbart.

2. Um entscheidende Urteile über Mirsä Schäfys Schaffen abzugeben, muss man seine aserbaidschanischen und persischen Gedichte finden. Ohne Gedichte können die Forscher ihre Meinungen nur auf Grund der Übersetzungen äußern. Zu Mirsä Schäfys „Lieder“n gehören die Gedichte über Süleyxa und Yusif, Tbilisi, Klagegedichte, über Hafiza, über Liebegedichte, über Besingung der weltlichen Überflusses und Wein, tröstliche Lieder, Zypresse. 
3. Obwohl Mirsa Schäfys Wazeh literarischen Schaffen den persischen Dichters Hafiz als seinen Lehrer nannte, fühlte man aber in seinen Werken den Geist von Fizuli. In diesem Sinne soll man Mirsä Schäfy Wazeh in der Literatur und Poesiewelt als Fizulis Nachfolger bewerten. Unter den Mirsä Schäfys Gedichten gibt es die Muster mit satirischem Inhalt. In manchen bestimmten Mustern kritisierte man die religiösen Menschen, die vom Weg der Wahrheit weit waren. Deshalb gefielen den religiösen Menschen Mirsa Schäfy nicht.

4. In seinen Gedichten benutzte er oft die Wörter wie Blumen, Wein, Schatz, Liebe, Mahbub, deshalb hasste ihn die Geistliche. Eigentlich konnten die Gläubiger nicht verstehen, dass diese Sprüche in der vernünftigen Literatur gebräuchliche Muster waren. In diesen Worten besang Mirsä Schäfy mehr die göttliche Liebe als die Weltliche. Das Besingen der göttlichen Liebe war als Mittel des Ausdrucks des Reichtums der menschlichen Ideenwelt. Seit 1840 begann Mirsä Schäfy Wazeh in Tbilisi im russischen Gymnasium mit der Ausbildung der aserbaidschanischen Sprache sich zu beschäftigen. In Gymnasien, die im Kaukasus geöffnet wurden, unterrichtete man in den Aserbaidschanischen und persischen Sprachen.

5. Deshalb benötigte man die besonderen Lehrbücher, die in diesen Sprachen geschrieben waren. Abbasgulu Aga Bakichanovs Werk „Qanuni Qüdsi“" benutzte man für die Ausbildung der persischen Sprache. Für die aserbaidschanische Ausbildung gab es kein solches Mittel.

6. Deshalb fasste Mirsä Schäfy mit Ivan Grigorjevitsch, der ein Student von Mirsa Kasym war und bei ihm Persisch gelernt hatte, zusammen eine Anthologie über die aserbaidschanische Sprache ab. Der
Wortschatzteil der Anthologie war schon im Januar 1851, aber selbst die Anthologie im April fertig. Die Anthologie „Tatarische Anthologie der aserbaidschanischen Adverbien“ bestand aus zwei Teilen. Der erste Teil hieß „Ganze und breite Anthologie der aserbaidschanischen Adverbien" und der zweite Teil aber „Extraktion aus der breiten Anthologie“.

7. In manchen Quellen nannte man dieses zusammen vorbereitetes von Mirsä Schäfy und des Oberlehrers im Gymnasium Ivan Grigorjevitsch „Buch von Turki“, „Anthologie Turki und zusammen abgefasste Anthologie von Mirsä Schäfy Sadyk oglu und dem Lehrer Grigorjevitsch des aserbaidschanischen Mundarts“.

8. Der erste von ihnen war für die hohen und zweite für die unteren Klassen und die Gebietsschulen abgefasst. Der zweite Teil mit dem Wörterbuch wurde im Jahre 1855 nach dem Tode M. Schäffy von Ivan Grigorijev in Tabris veröffentlicht und für eine lange Zeit in den unteren Klassen und in den Gebietschulen als Lehrbuch für das Erlernen Aserbaidschanisch gebraucht.

9. Die voll erweiterte Variante des ersten Teils der Literaturrichtung am Ende des Jahres 1856 veröffentlicht und ins Bildungsministerum und um es eine Zusage über den Gebrauch dieses Buches als Lehrbuch Erlaubnis gegeben. Aber einige Professoren der Peterburger Universität gaben eine negative Meinung über dieses Werk. Die Anthologie wird außerdem Wörterbuch noch in drei Teilen geteilt. Erster Teil besteheht aus den Artikeln, die aus persischen und arabischen Quellen ins Aserbaidschanisch übersetzen worden.

\section{References:}

1. (1875) Aus dem Nachlasse Mirza Schaffy. Berlin, 1875.

2. (1851) Die Lieder des Mirza Schaffy. Berlin.

3. Ohmədov FM (2007) Gəncənin tarix yaddaşı. Gəncə: Elm.

4. Ofəndiyev RS (1966) Azarbaycanın badii sənətkarlı̆̆l. Bak1: Azərnəşr.

5. Oliyeva NY (2013) Mirzə Şəfi Vazeh tadqiqatlarında Şərq və Qərb təfəkkürünün vahdati. Gəncə: Elm, 231 p.
6. Guliyeva NM, Häsänov EL (2014) Die traditionelle Gändschänischen Teppiche von Zeitraum der Aserbaidschanischen Gelehrten und Dichter Mirsä Schäfi Waseh als ethnoanthropologische quelle (XIX Jahrhundert). European Applied Sciences, 2: 3-5.

7. Hasanov EL (2013) Innovative approach to teaching and research of some traditional craft branches of Ganja of the end of XIX century. International Journal of Experimental Education 2: 81-83. 
8. Hasanov EL (2015) Aspetti Culturali della Ricerca delle Tradizioni Artigianali di Ganja dei Secoli XIX-XX. Mediterranean Journal of Social Sciences, vol. 6, № 2, part 1, pp. 372375. doi:10.5901/mjss.2015.v6n2s1p372

9. Həmidova İ (2000) Azərbaycan parça sənətinin tarixi inkişaf yolları. Elmi axtarışlar, VIII toplu, Bakı.
10. The dawn of Art (1974) Leningrad: Aurora Art Publishers.

11. (1901) Zeitschrift für Ethnorogie-Verhandlunge der Berliner Gesellschaft für Anthropologie, Ethnologie und Urgeschichte. Berlin, 1901, 8284.

EInur Lätif oglu Häsänov

Die Aserbaidschanische Nationale Wissenschaftliche Akademie

Die Gändschänischen Wissenschaftlichen Abteilung (Filiale), Ph.D. Doktorand, Korrespondierendes Mitglied der Internationalen

Akademie der theoretischen und angewandten Wissenschaften

Gändschä, Aserbaidschan 1-hasan@hotmail.com 